\title{
NEGATIVE FIR-PHOTOCONDUCTIVITY IN n-GaAs
}

\author{
W. Heisel, W. Bohm, and W. Prettl \\ Institut für Angewandte Physik, \\ Universität Regensburg, 8400 Regensburg, \\ W-Germany
}

Received December 29, 1980

Negative photoconductivity in n-GaAs has been observed in the far infrared spectral range between 20 and $29 \mathrm{~cm}^{-1}$. Negative photoconductivity occurs when a magnetic field is applied to the samples and impact ionization of shallow donors by the electric bias field is the dominant mechanism of electron excitation to the conduction band. A conceivable model qualitatively explaining the experimental results is proposed, which involves optical transitions from the lowest Landau subband to higher bound states of shallow donors.

Key words: semiconductors, epitaxial GaAs, far-infrared magnetospectroscopy, impact ionization, photoconductivity.

\section{Introduction}

The far-infrared (FIR) photoconductivity of high purity $n$-GaAs has been extensively investigated and has yielded detailed information about shallow donor states in this material $(1,2)$. The binding energy of shallow donors is $5.7 \mathrm{meV} \triangleq 45.6 \mathrm{~cm}^{-1}$. At helium temperature most electrons are bound to shallow donors. They can be excited to the conduction band by impact ionization by free carriers (3). This yields a strong increase of the current, possibly over several orders of magnitude, at a critical electric breakdown field of a few volts/cm depending on impurity 
concentration, compensation ratio, magnetic field and temperature (4). Normally photoconductivity measurements have been performed with electric bias fields well below the threshold of impact ionization. In this paper we report on investigations of the FIR-photoconductivity of n-GaAs in the region of impact ionization break-down of the current voltage characteristic as a function of an external magnetic field and optical power. Our results show that under these conditions negative photoconductivity can occur, i.e. the voltage across the sample increases under illumination in contrast to usual photoconductivity.

\section{Experimental}

The experiments were carried out using FIR molecular lasers with frequencies of $20.2 \mathrm{~cm}^{-1}\left(\mathrm{CH}_{3} \mathrm{~F}\right.$-laser $), 23.8 \mathrm{~cm}^{-1}$ ( $\mathrm{HCOOH}$ ) and $29.7 \mathrm{~cm}^{-1}$ ( $\mathrm{HCN}$ ). The samples were epitaxial n-GaAs layers on semiinsulating substrates with ohmic point contacts. All together five different samples were investigated with donor concentrations $\mathbb{N}_{D}=1 \ldots 10 \times 10^{-14} \mathrm{~cm}^{-3}$ and compensation ratios of the order of $50 \%$. In all cases negative photoconductivity could be observed. The measured results shown in the subsequent figures are taken from one sample with the $29.7 \mathrm{~cm}^{-1}$ line of the HCN-Iaser. They are typical for all investigated n-GaAs layers. The characteristics of this material are: $\mathbb{N}_{D}=7.2 \times 10^{14} \mathrm{~cm}^{-3}$, compensation ratio $\mathrm{N}_{\mathrm{A}} / \mathrm{N}_{\mathrm{D}}=0.49$, mobility $\mu=81.5 \times 10^{3} \mathrm{~cm}^{2} / \mathrm{Vs}$ at $77 \mathrm{~K}$; thickness $20 \mu \mathrm{m}$, size $3 \times 4 \mathrm{~mm}^{2}$.

The samples were mounted in a metallic light pipe at the center of a superconducting solenoid and immersed in liquid helium. Magnetic fields up to $B=3.8 \mathrm{~T}$ were applied. The measurements were performed in Faraday configuration with the electric bias field perpendicular to the magnetic field. Photoconductivity was measured as the change of the voltage across the sample with standard lock-in technique. The bias voltage was supplied either by a constant current source or by a load resistor.

\section{Results}

The photoconductive signal as a function of the magnetic field B for three different currents $I$ is shown in Fig. 1. For $I=1 \mu \mathrm{A}$ the electric bias field is below 


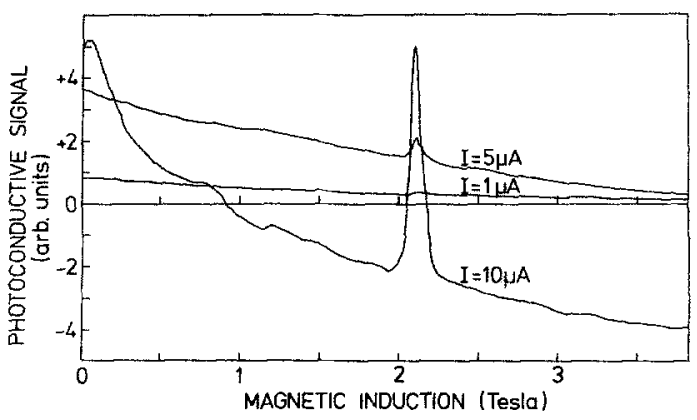

Figure 1. Photoconductive signal at $29.7 \mathrm{~cm}^{-1}$ as a function of the magnetic field at constant laser power for various currents $I . \quad I=1 \mu \mathrm{A}$ : Below impact ionization breakdown, $I=5 \mu \mathrm{A}$ and $10 \mu \mathrm{A}$ : above breakdown.

the impact ionization threshold in the whole range of the magnetic field. The photoconductivity is positive in this case, i.e. the voltage change across the sample is negative. As the laser frequency of $29.7 \mathrm{~cm}^{-1}$ falls into the low frequency wing of the $1 \mathrm{~s}-2 \mathrm{p} \ldots$._. Ine and the corresponding transition energy is almost independent of the magnetic field (5), the positive photoconductivity is due to the photothermal ionization process which produces a strong peak in the photoconductivity spectrum as the $1 \mathrm{~s}-2 \mathrm{p}$ transition frequency (6). If the bias voltage equals the critical value of impact ionization breakdown the signal decreases with increasing magnetic field, crosses zero and becomes negative. This is shown by the $I=10 \mu \mathrm{A}$ curve in Fig. 1. At zero magnetic field the photoconductivity was found to be positive for all currents. At the frequencies of $20.2 \mathrm{~cm}^{-1}$ and $23.8 \mathrm{~cm}^{-1}$ the photo response showed a very similar behaviour as displayed in Fig. 1 for $29.7 \mathrm{~cm}^{-1}$. Therefore we do not present the measurements here. In these cases the positive photo response below the impact ionization breakdown must be due to transitions from thermally exited donor states to the conduction band, because the photon energy is too small to excite $1 \mathrm{~s}-2 \mathrm{p}-$ transitions. The narrow line at $\mathrm{B}=2.1 \mathrm{~T}$ (Fig. 1), which strongly increases with current, results from cyclotron resonance of free electrons. It has been shown that the 
main contribution to the change in conductivity of n-GaAs under cyclotron resonance conditions is due to increasing free carrier density by the redistribution from donor states to the optically depleted Landau levels and not due to a change in mobility (7). Therefore the strength of the cyclotron resonance line reflects the growing concentration of free electrons excited by impact ionization of shallow donors. The photo response as a function of the current and the voltage current characteristic for zero magnetic field is shown in Fig. 2. The signal increases at low currents proportional to bias voltage showing the usual behaviour of a photo conductor. Above the impact ionization threshold the signal decreases to a low but positive value due to the depletion of neutral donors by impact ionization. In Fig. 3 the current dependence of the response and the voltage current characteristics are shown for $\mathrm{B}=3 \mathrm{~T}$. The photosignal is shown for two laser powers: $\sim 2 \mathrm{~mW}$ and $\sim 100 \mathrm{~mW}$. Below the impact ionization breakdown the response is analogous to the case of $B=0$. Above the breakdown the behaviour of the photosignal depends in a nonlinear way on laser power. The $2 \mathrm{~mW}$ curve decreases and becomes negative in the flat region of the $\mathrm{V}-\mathrm{C}$ characteristic. The signal at $100 \mathrm{~mW}$ laser power also decreases but it remains positive in the current range of Fig. 3. It changes also sign at a higher current not shown in Fig. 3.

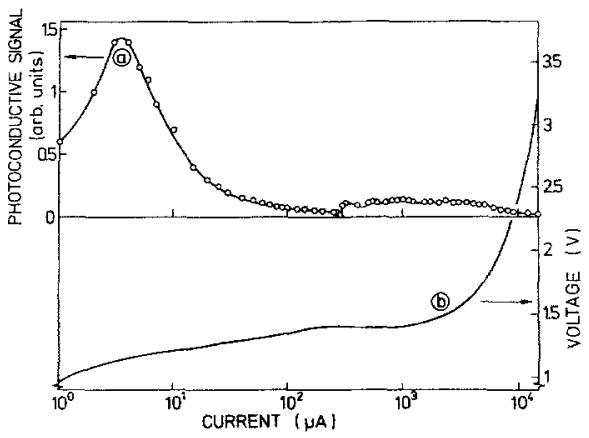

Figure 2. Photoconductive signal (a) and voltage across the sample (b) as a function of the current at $B=0$. 


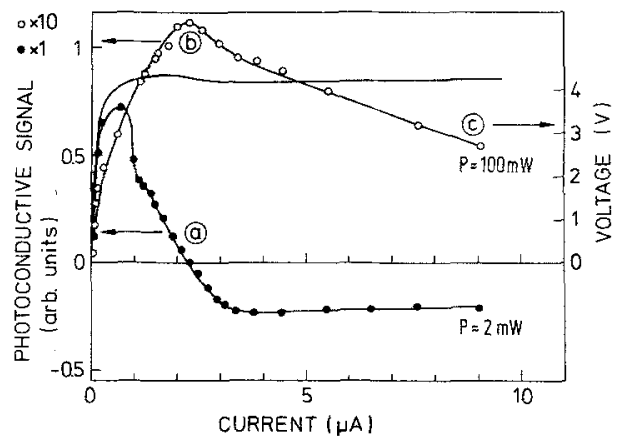

Figure 3. Photoconductive signal (a) and (b) and bias voltage (c) as a function of the current at $B=3 \mathrm{~T}$. Optical power (a): $\sim 2 \mathrm{~mW},(\mathrm{~b}): \sim 100 \mathrm{~mW}$.

The peculiar behaviour of the photoconductive response upon laser power close to the impact ionization breakdown has been measured and is shown in Fig. 4. The relative change in the conductivity $\Delta \sigma / \sigma$ at $B=3 \mathrm{~T}$ is plotted for various bias voltages as a function of optical power. Well below the breakdown the response is positive. Approaching the threshold the signal becomes negative at low power and turns to positive photoconductivity with increasing laser power. The depth of the minimum of the response increases with the bias voltage. These measurements show that close to the breakdown positive and negative photoconductivity may occur at the same biasing conditions depending on the laser power. Fig. 5 shows an analogous measurement at $I=50 \mu \mathrm{A}$, well in the flat portion of the voltage current curve. In this case the photoconductivity is negative in the whole power range.

\section{Discussions}

Negative photoconductivity has been observed in many materials for optical interband excitations and ionizing transitions of deep impurity levels. It is not a unique physical phenomenon. For different materials different models have been developped to explain the occurrence of negative photoconductivity $(8,9)$. Our experimental results on $n$-GaAs indicate that the presence of a magnetic field and impact ionization as the dominant process of carrier 


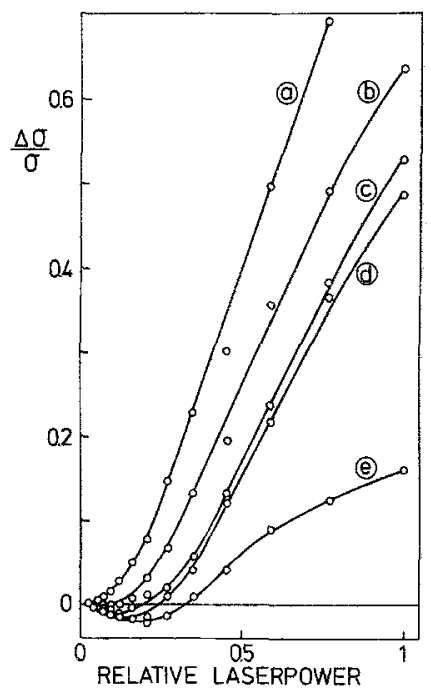

Figure 4. Relative photoconductive signal $\Delta \sigma / \sigma$ at $B=3 \mathrm{~T}$ for various currents $I$ and voltages $U$ across the sample below impact ionization breakdown. Breakdown voltage: $\mathrm{U}_{\mathrm{B}}=4.3$ Volts. Power $=1$ corresponds to about $100 \mathrm{~mW}$. $\mathrm{a}: I=0.37 \mu \mathrm{A}, U=3.68 \mathrm{~V} ; \mathrm{b}: I=0.7 \mu \mathrm{A}, U=4.05 \mathrm{~V}$; $c: I=0.94 \mu \mathrm{A}, U=4.13 \mathrm{~V} ; \mathrm{d}: I=1.03 \mu \mathrm{A}, U=4.25 \mathrm{~V}$; e: $I=1.72 \mu \mathrm{A}, U=4.3 \mathrm{~V}$.

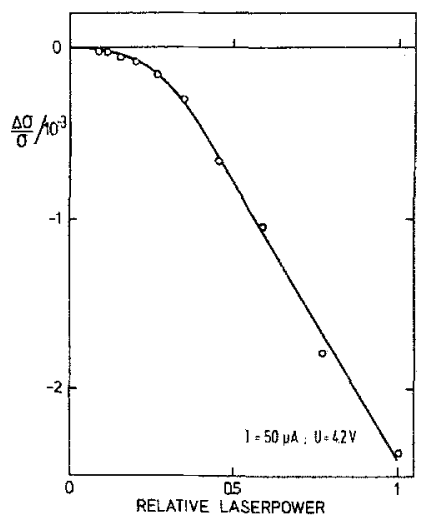

Figure 5. Relative photoconductive signal $\Delta \sigma / \sigma$ at $B=3 \mathrm{~T}$ above impact ionization breakdown. Power $=1$ corresponds to about $100 \mathrm{~mW}$. 
excitation is necessary to observe negative photoconductivity. The requirement $B \neq 0$ rules out a mobility effect. Heating the free electrons by FIR radiation reduces the mobility if electron phonon scattering predominantly determines the momentum relaxation (10). This contribution to negative photoconductivity, however, should also be effective at $B=0$, in contrast to our observations. In the following discussion we propose a conceivable model which involves optical excitations of electrons from the lowest Landau continuum to higher bound states of shallow donors. This process reduces the concentration of free carriers and should be particularly efficient if there is a large excess of electrons in the $\mathrm{N}=0$ Landau band due to impact ionization.

The magnetic field splits the conduction band into Landau subbands and splits and shifts the donor levels. Metastable shallow donor bound states arise above the low energy edge of the $N=0$ Landau continuum $(11,12)$. Such states have lifetimes comparable to shallow donor states below the $\mathrm{N}=\mathrm{O}$ Landau band as can be deduced from photoconductivity spectra (see $e \cdot g$. the $1 \mathrm{~s} \rightarrow 2 p_{+}$transition at $B=2.99 \mathrm{~T}$ in Ref.(4). We suppose that the negative contribution to the photoconductivity results from optical transition from the lowest Landau continuum to higher lying shallow donor states which reduce the concentration of free electrons. This process is in competition with ionizing transitions of excited states and the photo thermal ionizing process in connection with $1 \mathrm{~s}-2 \mathrm{p}$ - transitions for $29.7 \mathrm{~cm}^{-1}$. We denote the photoinduced change of the free electron concentration by $\Delta \mathrm{n}=\Delta \mathrm{n}_{+}+\Delta \mathrm{n}_{-}$, where $\Delta \mathrm{n}_{+}$and $\Delta \mathrm{n}_{-}$are the contributions due to the mechanisms yielding positive and negative photosignals respectively. At low laser powers $\Delta n_{+-}$becomes $\Delta n_{+} \propto N_{D}-N_{A}-n$ and $\Delta n_{-} \propto-n$, where $n$ is the electron density in the lowest Landau band. At helium temperatures and electric fields well below the impact ionization threshold $n<<N_{D}-N_{A}$, and $\left|\Delta n_{-}\right|<<\Delta n_{+}$, photoconductivity is positive. Increasing the electric field close to but yet below the threshold magnitude strongly increases $\mathrm{n}$ due to impact ionization. Therefore $\left|\Delta \mathrm{n}_{-}\right|$may exceed $\Delta \mathrm{n}_{+}$ and thus negative photoconductivity occurs. The photo induces electron densities saturate at high optical power as $\Delta \mathrm{n}_{+}=\mathrm{N}_{\mathrm{D}}-\mathrm{N}_{\mathrm{A}}$ and $\Delta \mathrm{n}_{-}=-\mathrm{n}$. Thus as long as $\mathrm{n}<\mathrm{N}_{D^{-}} \mathrm{N}_{\mathrm{A}}$ at high laser power positive photoconductivity may appear again. This consideration qualitatively explains the photosignal as a function of the laser power displayed in Fig. 4 . If 
the electric field is increased further up to the sustaining field of the impact ionization breakdown, $n$ approaches $\mathbb{N}_{D}-\mathbb{N}_{A}$ and $\Delta \mathrm{n}_{+}$vanishes accordingly. Therefore the photosignal at high current in the constant voltage portion of the current voltage characteristic is negative for all laser powers as experimentally observed (see Fig. 5). The saturation value of $\Delta n_{-}$now becomes $\Delta n_{-}=-n \simeq-\left(N_{D}-N_{A}\right)$ and cannot be reached by the laser used in our experiments. An elaboration of this model and a detailea analysis of our measurement will be presented in another publication.

\section{Acknowledgments}

We acknowledge valuable discussions with G. Bauer, E. Schöll, and W. Zawadzki. We are indebted to E. Bauser, Max-Planck-Institut für Festkörperforschung, Stuttgart, who placed the GaAs samples to our disposal.

\section{References}

1. For a review see: G.E. Stillman, C.M. Wolfe, and J.O. Dimmock in: Semiconductors and Semimetals, ed. R.K. Willardson and A.C. Beer, vol. 12, pp. 169, Academic Press, 1977.

2. M.S. Skolnick, A.C. Carter, Y. Couder, and R.A. Stradling, J.Opt.Soc.Am. 67, 947 (1977).

3. D.J. Oliver, Phys.Rev. 127, 1045 (1962).

4. R.A. Reynolds, Solid State Electron. 11, 385 (1968).

5. G.E. Stillman, D.M. Larsen, C.M. Wolfe, and R.C. Brand, Solid state Commun. 2, 2245 (1971).

6. G.F. Stillman, C.M. Wolfe, and J.o. Dimmock, Proc.Int. Conf. on Phys.Semicond., Warsaw (1972), p. 863.

7. H.J.A. Bluyssen, I.C. Maan, I.J. van Ruynen, F. Williams, and P. Wyder, Solid State Commun. 25, 895 (1978).

8. F. Stöckmann, Z.Phys. 143, 348 (1955).

9. S.G. Petrosyan and A.Ya. Shik, Sov.Phys. Semicond. 10, 804 ( 1976. 
10. A. Philipp, F. Kuchar, and K. Seeger, phys.stat.sol.(b) 79, $115(1977)$.

11. D.M. Larsen, Phys.Rev. B 8, 535 (1973).

12. E.M. Gershenzon, G.N. Gol'tsman, and A.I. Elat'ev, Sov. Phys. JETP 45, 555 (1977). 